\title{
FK866-induced NAMPT inhibition activates AMPK and downregulates mTOR signaling in hepatocarcinoma cells
}

\author{
Susanne Schuster ${ }^{a,{ }^{*}, 1}$, Melanie Penke ${ }^{a, 1}$, Theresa Gorski ${ }^{a}$, Rolf Gebhardt ${ }^{b}$, \\ Thomas S. Weiss ${ }^{c}$, Wieland Kiess ${ }^{a}$, Antie Garten ${ }^{a}$ \\ a Center for Pediatric Research Leipzig, University Hospital for Children and Adolescents, Faculty of Medicine, University of \\ Leipzig, Liebigstr. 21, 04103 Leipzig, Germany \\ b Institute of Biochemistry, Faculty of Medicine, University of Leipzig, Johannisallee 30, 04103 Leipzig, Germany \\ c Children's University Hospital, University of Regensburg, Franz-Josef-Strauss-Allee 11, 93053 Regensburg, Germany
}

\section{Article Info}

Article history:

Received 15 January 2015

Available online 3 February

2015

Keywords: NAMPT FK866

NAD AMPK mTOR

Hepatocarcinoma cells

\begin{abstract}
Background: Nicotinamide phosphoribosyltransferase (NAMPT) is the key enzyme of the NAD salvage pathway starting from nicotinamide. Cancer cells have an increased demand for NAD due to their high proliferation and DNA repair rate. Consequently, NAMPT is considered as a putative target for anti-cancer therapies. There is evidence that AMP-activated protein kinase (AMPK) and mammalian target of rapamycin (mTOR) become dysregulated during the development of hepatocellular carcinoma (HCC). Here, we investigated the effects of NAMPT inhibition by its specific inhibitor FK866 on the viability of hepatocarcinoma cells and analyzed the effects of FK866 on the nutrient sensor AMPK and mTOR complex1 (mTORC1) signaling.

Results: FK866 markedly decreased NAMPT activity and NAD content in hepatocarcinoma cells (Huh7 cells, Hep3B cells) and led to delayed ATP reduction which was associated with increased cell death. These effects could be abrogated by administration of nicotinamide mononucleotide (NMN), the enzyme product of NAMPT. Our results demonstrated a dysregulation of the AMPK/mTOR pathway in hepatocarcinoma cells compared to non-cancerous hepatocytes with a higher expression of mTOR and a lower AMPKa activation in hepatocarcinoma cells. We found that NAMPT inhibition by FK866 significantly activated AMPKa and inhibited the activation of mTOR and its downstream targets p70S6 kinase and 4E-BP1 in hepatocarcinoma cells. Non-cancerous hepatocytes were less sensitive to FK866 and did not show changes in AMPK/mTOR signaling after FK866 treatment.

Conclusion: Taken together, these findings reveal an important role of the NAMPT-mediated NAD salvage pathway in the energy homeostasis of hepatocarcinoma cells and suggest NAMPT inhibition as a potential treatment option for HCC.
\end{abstract}

\footnotetext{
Abbreviation: NAMPT, nicotinamide phosphoribosyltransferase; AMPK, AMP- activated protein kinase; mTOR, mammalian target of rapamycin; HCC, hepatocel- lular carcinoma; PHH, primary human hepatocytes; mTORC1, mTOR complex1; NMN, nicotinamide mononucleotide; p70S6 kinase, 705 ribosomal protein S6 ki- nase; $4 \mathrm{E}-\mathrm{BP1}$, eukaryotic initiation factor $4 \mathrm{E}$ binding protein 1; NAD, nicotinamide adenine dinucleotide; PARPs, poly(ADPeribose) polymerases; MARTs, mono-ADP ribosyltransferases; $\mathrm{PI}$, propidium iodide; ATP, adenosine triphosphate; EDTA, ethylenediaminetetraacetic acid; TSA, trichostatin A; GAPDH, glyceraldehyde-3- phosphate dehydrogenase; cpm, counts per minute.

* Corresponding author. Fax: +493419726069.

E-mail address: Susanne.Schuster@medizin.uni-leipzig.de (S. Schuster).

1 Both authors contributed equally to this work.
}

1. Introduction

The co-factor nicotinamide adenine dinucleotide (NAD) plays a crucial role in multiple cellular processes and is substrate for a variety of enzymes and regulatory proteins [1]. In humans a main portion of NAD is generated via the nicotinamide (NAM) salvage pathway, in which nicotinamide phosphoribosyltransferase (NAMPT) catalyzes the rate-limiting step in the biosynthesis of NAD yielding nicotinamide mononucleotide (NMN) $[2,3]$. As NAD is rapidly consumed in cells $(1 \mathrm{~h})$ and converted to NAM [4], NAMPT is essential for the replenishment of the intracellular NAD pool. The development of many cancers is associated with increased NAMPT expression [5]. Cancer cells have a high rate of NAD turnover due to 
Published in final edited form as: Biochemical and Biophysical Research Communications 458 (2015) 334e340,

http://dx.doi.org/10.1016/j.bbrc.2015.01.111

their increased energy demand and a high activity of NADdependent enzymes, such as poly (ADP-ribose) polymerases (PARPs), mono-ADP ribosyltransferases (MARTs) and sirtuins, required for DNA repair, genome stability and proliferation $[1,5]$. Therefore, cancer cells are more susceptible to NAMPT inhibition than normal cells $[6,7]$. In previous studies, we found that NAMPT is released from hepatocytes [8] as well as differentially expressed and more enzymatically active in hepatocarcinoma cells compared to non-cancerous human hepatocytes [9]. Hepatocellular carci- noma (HCC) is the second leading cause of cancer-related deaths [10]. The only available proven systemic therapy for HCC is the multi-targeting kinase inhibitor sorafenib [11]. An effective second- line agent for patients with sorafenib failure or intolerance has yet to be identified. This has led to an intensive search for molecular pathways and novel compounds for the treatment and prevention of HCC. Targeting NAMPT activity and intracellular NAD content represents a novel therapeutic concept for HCC. The specific NAMPT inhibitor FK866 is a competitive inhibitor that was selected by an anticancer screening system differentiating acute cytotoxicity from growth inhibition $[12,13]$. FK866 has been evaluated in a broad variety of tumors, including solid tumors and leukemia [14e16] in vitro and in nude mouse xenografts [17e19], where FK866 was able to reduce or attenuate tumor growth.

In HCC tissue, AMP-activated protein kinase (AMPK), a major regulator of cellular energy homeostasis that coordinates multiple metabolic pathways, has been shown to be dysregulated compared to normal tissue $[20,21]$. AMPK activity opposes tumor develop- ment and negatively regulates the Warburg effect (aerobic glycol- ysis) leading to suppression of tumor growth in vivo [20e22]. AMPK translates changes in glucose availability and fluctuation of energy to mammalian target of rapamycin (mTOR) and thereby acts as a master energy sensor to modulate cellular activities in response to energy stress $[23,24]$. mTOR, a serine/threonine protein kinase, has been observed to be increased in multiple human cancers, including HCC, where it is associated with less differentiated tu- mors, earlier tumor recurrence, and worse survival outcomes $[25,26]$. Inhibition of mTOR has proven efficacious in clinical trials $[26,27]$. Recently, there is great scientific interest in finding mo- lecular pathways and novel compounds that target $\mathrm{AMPK} / \mathrm{mTOR}$ signaling as a new treatment option for $\mathrm{HCC}$.

Little is known about the interaction of NAMPT and AMPK/ mTOR signaling during the development of HCC. In this study, we investigated the effects of the NAMPT inhibitor FK866 on hep- atocarcinoma cells and non-cancerous human hepatocytes. We asked whether or not FK866-induced energy stress might activate AMPK and modify the mTOR signaling pathway and whether the observed effects could be rescued by the NAMPT enzyme product NMN.

2. Material and methods

\subsection{Material}

Cell culture media, supplements and antibiotics were obtained from PAA (Cölbe, Germany) or Invitrogen (Karlsruhe, Germany). FK866, nicotinamide mononucleotide (NMN) and camptothecin were purchased from SigmaeAldrich (Munich, Germany). Etoposide was purchased from Merck Millipore (Darmstadt, Germany).

\subsection{Hepatocarcinoma cell lines}

Huh7 cells (p53-mutated) and Hep3B cells (p53-deficient) were maintained in DMEM medium with high glucose or MEM medium, respectively. Media were supplemented with 10\% fetal bovine serum (FBS), $2 \mathrm{mM}$ glutamine, $100 \mathrm{IU}$ penicillin and $100 \mathrm{mg} / \mathrm{mL}$ streptomycin. All cells were grown at $37^{\circ} \mathrm{C}$ in a humidified atmosphere of $95 \%$ air and $5 \% \mathrm{CO}_{2}$.

\subsection{Primary human hepatocytes}

Tissue samples from patients undergoing liver surgery at the University Medical Center Regensburg were used. Primary human hepatocytes $(\mathrm{PHH})$ were isolated and cultivated as described recently [28]. Briefly, non-neoplastic tissue samples from liver re- sections were obtained from patients undergoing partial hepatec- tomy for metastatic liver tumors of colorectal cancer. PHHs were isolated using a modified two-step EGTA/collagenase perfusion procedure and plated on collagen coated dishes. Experimental procedures were performed according to the guidelines of the charitable state controlled foundation HTCR (Human Tissue and Cell Research, Regensburg, Germany), with the informed patient's consent approved by the local ethical committee of the University of Regensburg. All experiments involving human tissues and cells have been carried out in accordance to The Code of Ethics of the World Medical Association (Declaration of Helsinki). Cells were seeded in Williams' Medium E containing $2 \mathrm{mM}$ glutamine, $10^{-7} \mathrm{~mol} / \mathrm{L}$ dexamethasone, $100 \mathrm{IU}$ penicillin, 100 $\mathrm{mg} / \mathrm{mL}$ streptomycin and $10 \% \mathrm{FBS}$. All cells were grown at 37 ${ }^{\circ} \mathrm{C}$ in a humidified atmo- sphere of $95 \%$ air and $5 \% \mathrm{CO}_{2}$.

\subsection{Cell treatments}

FK866 was dissolved in DMSO to create a stock solution of $10 \mathrm{mM}$. NMN was dissolved in the appropriate medium for a stock solution of $100 \mathrm{mM}$. After $16 \mathrm{~h}$ serum starvation, cells were treated with the indicated concentration of FK866 alone or in combination with NMN [500 mM] for 24, 48 and $72 \mathrm{~h}$. 
Published in final edited form as: Biochemical and Biophysical Research Communications 458 (2015) 334e340,

http://dx.doi.org/10.1016/j.bbrc.2015.01.111

\subsection{Cell viability and apoptosis}

Cell viability analysis was conducted using the cell proliferation reagent WST-1 (Roche, Grenzach-Wyhlen, Germany) according to manufacturer's instructions. To examine the effects of FK866 on cell death, the number of dead cells was measured by FACS analysis at different time points $(48 \mathrm{~h}$, $72 \mathrm{~h}$ ) using the AnnexinV-FITC Apoptosis Detection Kit (BD Pharmingen ${ }^{\mathrm{TM}}$, Franklin Lakes, USA). Adherent and floating cells were analyzed according to manufac- turer's protocol. Samples were analyzed using a Beckton-Dickinson FACS LSRII. As positive control, apoptosis was induced via camptothecin [2 mM] and etoposide [85 mM] for 24 h. Annexin ${ }^{+}$ $\left(\mathrm{An}^{+}\right)$and

double-stained $\mathrm{An}^{+} /$propidium iodide $\left(\mathrm{Pl}^{+}\right)$cells were considered as dead cells.

\subsection{ATP measurement}

ATP levels were measured with the luminescent-based CellTiter-Glo ${ }^{\circledR}$ Luminescent Cell Viability Assay (Promega, Madison, USA) according to the manufacturer's protocol.

\subsection{Western Blot}

Protein extraction and Western Blot analysis were performed as described previously [9]. Primary antibodies used for immuno- blotting included anti-phospho-AMPKa(Thr 172), antiAMPKa, anti- phospho-mTOR(Ser2448), anti-mTOR, antitubulin, anti-phospho p70S6 kinase(Thr389), anti-p70S6 kinase, anti-phospho-4E- BP1(Ser65), anti-4E-BP1, antiacetylated lysine (Cell Signaling, Beverly, MA, USA) and antiGAPDH (MerckMillipore, Schwalbach, Germany). Appropriate secondary antibodies were purchased from DAKO (Hamburg, Germany). Immunoblotting for GAPDH or tubulin was performed to verify equivalent amounts of loaded protein. Densitometric analysis was performed using ImageJ 1.41 Software $(\mathrm{NIH}$, USA).

\subsection{NAMPT enzymatic activity}

NAMPT activity was measured by the conversion of ${ }^{14} \mathrm{C}$ labeled nicotinamide to ${ }^{14} \mathrm{C}$-NMN using a method previously described $[9,29]$. Radioactivity of ${ }^{14} \mathrm{C}-\mathrm{NMN}$ was quantified in a liquid scintillation counter in counts per minute (cpm) (Wallac 1409 DSA, PerkinElmer). NAMPT activity (cpm) was normalized to total protein concentration as measured by the BCA protein assay.

\subsection{NAD measurement}

Concentrations of NAD from whole-cell extracts were quantified by HPLC analysis using a SUPELCOSIL ${ }^{\mathrm{TM}}$ LC-18-T HPLC column (Sigma Aldrich) at a flow rate of $0.8 \mathrm{ml} / \mathrm{min}$ with $100 \%$ buffer A (potassium phosphate buffer $\mathrm{pH}$ 6.0) from 0 to $2 \mathrm{~min}$, a linear gradient to $85 \%$ Buffer $A / 15 \%$ Buffer B (100\% methanol) from 2 to $5 \mathrm{~min}, 85 \%$ Buffer $A / 15 \%$ Buffer $B$ from 5 to $10 \mathrm{~min}$, a linear gradient to $100 \%$ Buffer $A$ from 10 to 12 min and $100 \%$ Buffer $A$ from 12 to $15 \mathrm{~min}$. NAD was eluted as a sharp peak at $8 \mathrm{~min}$ and quantitated based on the peak area compared to a standard curve and normalized to total protein concentration as measured by the BCA protein assay. 
Published in final edited form as: Biochemical and Biophysical Research Communications 458 (2015) 334e340, http://dx.doi.org/10.1016/j.bbrc.2015.01.111

\section{Results}

3.1. FK866-induced NAMPT inhibition significantly decreased NAD levels in human hepatocarcinoma cells which could be ameliorated by NMN administration

We stimulated hepatocarcinoma cells with FK866 [10 nM] and found significantly reduced NAMPT activity $(-74.9 \pm 8.1 \%$ in Huh7 cells, $-38.1 \pm 3.7 \%$ in Hep3B cells) (Fig. 1 A) which caused a sharp decline of NAD levels (Huh7 cells $3.3 \pm 0.3$ $\mathrm{mmol} / \mathrm{g}$ protein [con] vs. $0.3 \pm 0.2 \mathrm{mmol} / \mathrm{g}$ protein [10 $\mathrm{nM}$ FK866]; Hep3B cells $2.2 \pm 0.7 \mathrm{mmol} /$ $g$ protein [con] vs. $0.2 \pm 0.08 \mathrm{mmol} / \mathrm{g}$ protein $[10 \mathrm{nM}$ FK866]) (Fig. 1B). Co-treatment with NMN restored intracellular NAD levels in all tested cell lines (Fig. 1B). To investigate the sensitivity of non- cancerous human hepatocytes towards FK866, we used the same treatment conditions as for hepatocarcinoma cells and found that non-cancerous hepatocytes showed no significant reduction in NAMPT activity and NAD levels at 10 nM FK866 after 48 h (Supplement Fig. 1A,B).

Emerging evidence suggests that the cellular acetylation state is associated with the energy state of a cell [30]. We could show that FK866-induced NAD depletion led to a decreased activity of NAD-
A

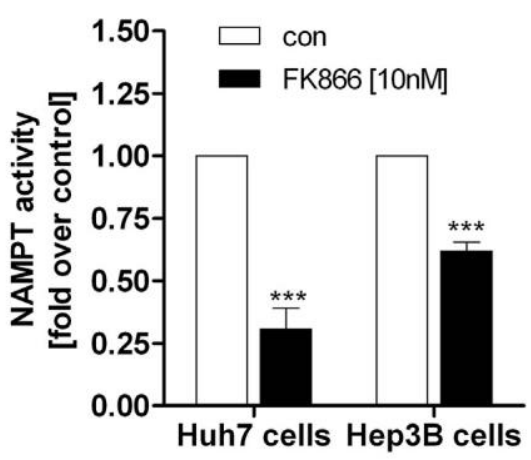

C

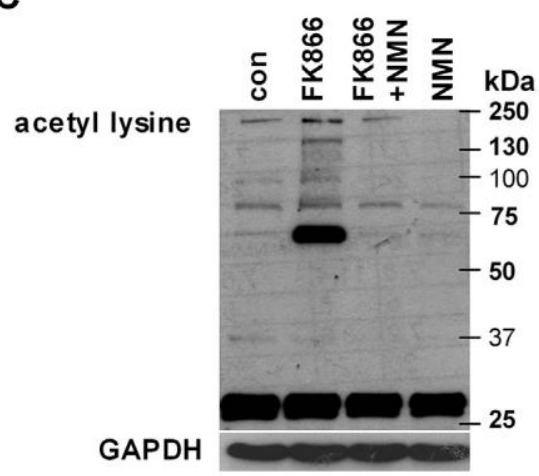

B
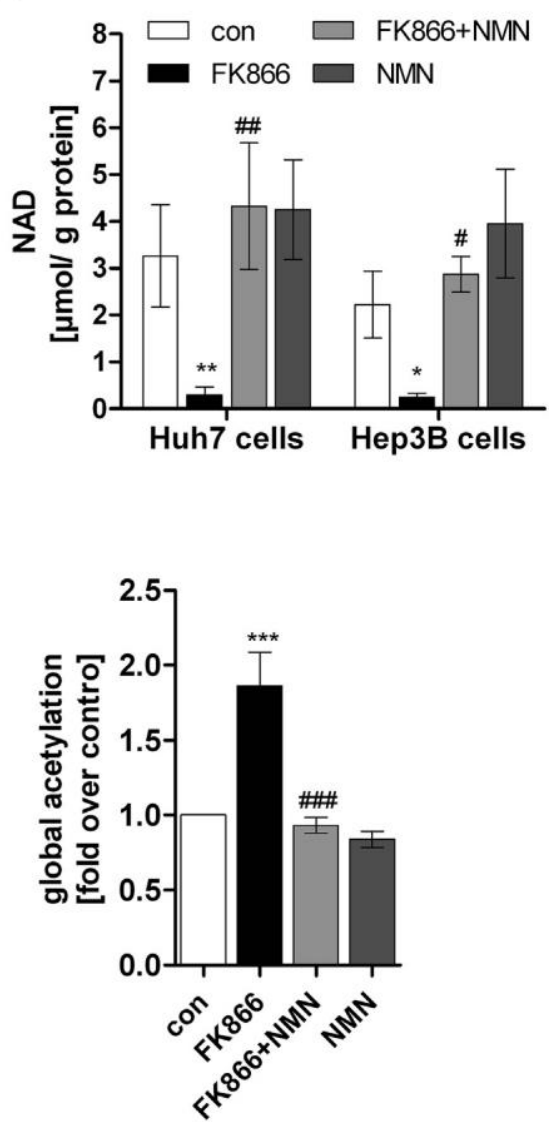

Fig. 1. FK866 reduced NAMPT activity and NAD content and increased global acetylation of lysine residues. A) NAMPT activity and B) NAD content were measured after $24 \mathrm{~h}$ and were normalized to total protein amount in each sample (n 1/4 3). C) Western Blot analysis of acetylated lysine residues in lysates of Hep3B cells treated with FK866 [10 nM], a combination of FK866 + NMN or NMN alone for $48 \mathrm{~h}$. GAPDH was used as loading control. Densitometric analysis of each lane was performed in four independent Western Blots (n 1/4 4). Cells stimulated with serum-free medium were used as control [con] and were set 1 . Data are represented as mean \pm SEM and statistical analysis was performed using one- way ANOVA and the Bonferroni post hoc test $\left({ }^{*} p<0.05,{ }^{* *} p<0.01,{ }^{* *} p<0.001\right.$ compared to serum-free medium control; \#p < 0.05, \#\#p < 0.01, \#\#\# < 0.001 compared to FK866 [10 nM]). 
Published in final edited form as: Biochemical and Biophysical Research Communications 458 (2015) 334e340, hitp://dx.doi.org/10.1016/i.bbrc.2015.01.111

A

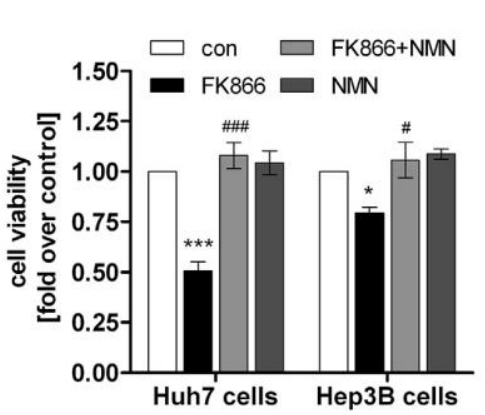

D

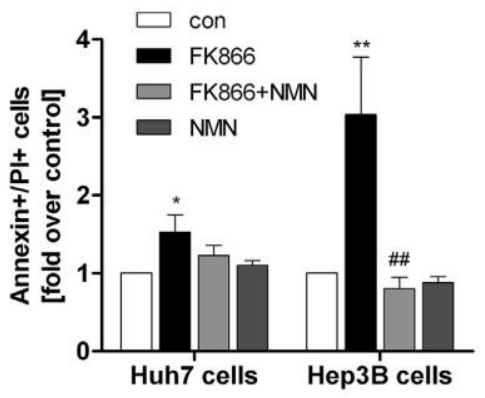

B

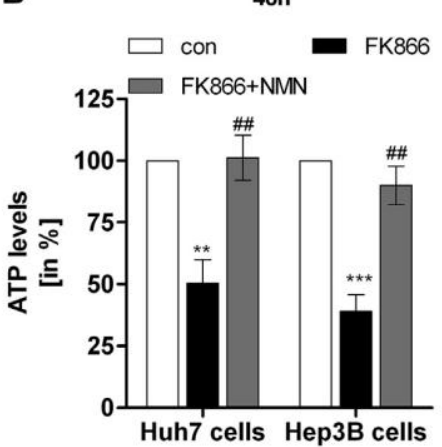

Huh7 cells
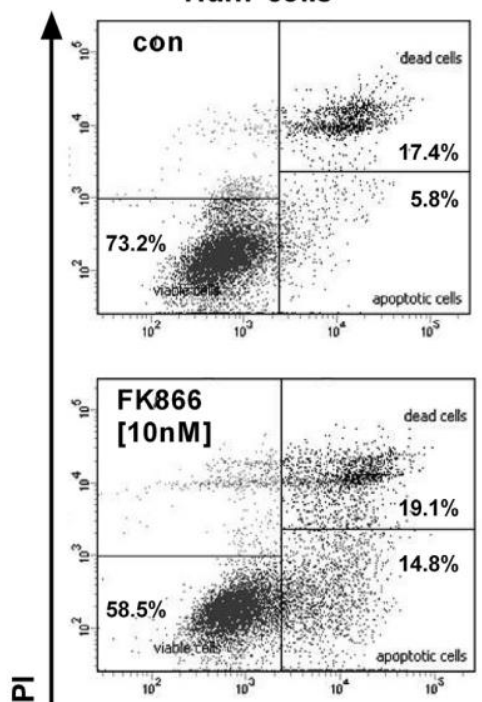

$\bar{\alpha}$
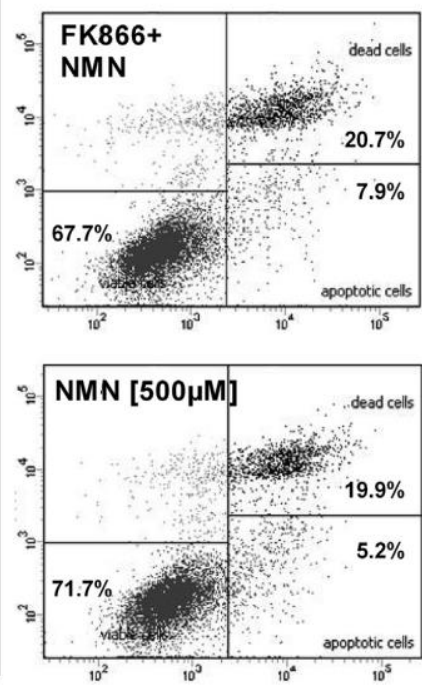

C

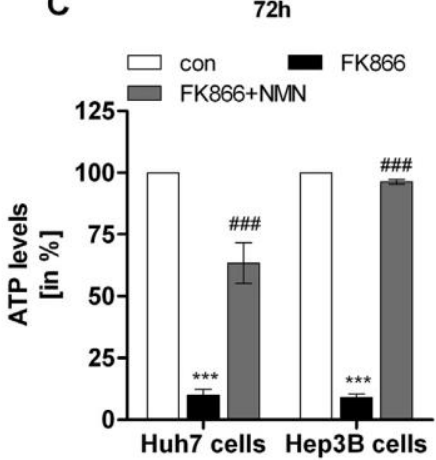

Hep3B cells
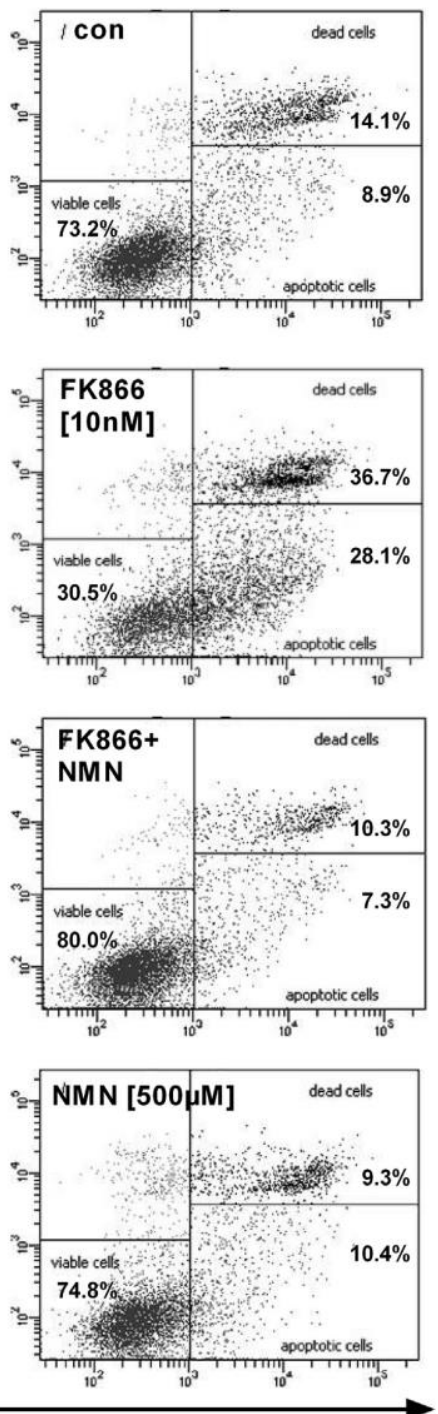

Annexin V-FITC

Fig. 2. FK866-induced NAMPT inhibition reduced cell viability and ATP content and induced delayed cell death in hepatocarcinoma cells. A) Cell viability of Huh7 and Hep3B cells after $24 \mathrm{~h}$ was measured using WST1-reagent (n 1/4 4). ATP content after B) $48 \mathrm{~h}$ and C) $72 \mathrm{~h}$ treatment with $10 \mathrm{nM} \mathrm{FK866} \mathrm{(n} \mathrm{1/43).} \mathrm{Cells} \mathrm{stimulated}$ with serum-free medium were used as control [con] and were set 100\%. D) AnnexinV-FITC/PI assay of Huh7 and Hep3B cells treated with FK866, FK866 + NMN or NMN alone for $72 \mathrm{~h}(\mathrm{n} 1 / 43)$. Cells stimulated with serum-free medium were used as control [con] and were set 1. Representative dot plots of the AnnexinV-FITC/PI staining in Huh7 and Hep3B cells are shown including the percentage of viable, $\mathrm{An}^{+}$and $\mathrm{An}^{+} / \mathrm{PI}^{+}{ }^{-}$cells. Data are represented as mean $\pm \mathrm{SEM}$ and statistical analysis was performed using one-way ANOVA and the Bonferroni post hoc test $\left({ }^{*} p<0.05,{ }^{* \star} p<0.01,{ }^{* *} p<0.001\right.$ compared to serum-free medium control; $\# p<0.05, \# \# p<0.01, \# \# p<0.001$ compared to FK866 [10 nM]). 
Published in final edited form as: Biochemical and Biophysical Research Communications 458 (2015) 334e340,

http://dx.doi.org/10.1016/j.bbrc.2015.01.111

dependent lysine deacetylases as measured by an increased global acetylation of lysine residues (+1.9-fold, $p<0.001$ ) (Fig. 1C). The administration of NMN abrogated the FK866induced hyper- acetylation of lysine residues $(p<0.001)$ (Fig. 1C).

\subsection{NAMPT inhibition by FK866 reduced cell}

viability, induced energy stress and led to delayed cell death in human hepatocarcinoma cells

We could detect a decreased cell viability in hepatocarcinoma cells $(-49.4 \pm 4.6 \%$ in Huh7 cells, $-20.6 \pm$ $2.8 \%$ in Hep3B cells) (Fig. 2A) after $24 \mathrm{~h}$ of FK866 treatment. We wanted to investigate whether FK866-induced NAD depletion would result in a reduction of ATP generation and therefore would induce cellular energy stress in hepatocarcinoma cells. Time course studies revealed that ATP levels were lowered in Huh7 cells $(-49.6 \pm 9.5 \%, p<0.01)$ and Hep3B cells $61.1 \pm 6.8 \%, p<0.001$ ) after $48 \mathrm{~h}$ of treatment with $10 \mathrm{nM}$ FK866 (Fig. 2B). The ATP levels further declined after $72 \mathrm{~h}$ in Huh7 cells $(-90.2 \pm 2.5 \%, p<0.001)$ and Hep3B cells $f$ $91.1 \pm 1.5 \%, p<0.001$ ) (Fig. 2C). The co-administration of NMN could ameliorate ATP levels in Huh7 and Hep3B cells after 48 and $72 \mathrm{~h}$ (Fig. 2B,C). After $72 \mathrm{~h}$, subsequent to the drop of NAD levels, the effects of FK866 on cell death became evident when measuring

$\mathrm{An}^{+} / \mathrm{PI}^{+}$-stained cells. Hep3B cells, a p53-deficient cell line, already

displayed an increase in $\mathrm{An}^{+} / \mathrm{Pl}^{+}$cells after $48 \mathrm{~h}$ of FK866 treatment $(+1.8$-fold, $p<0.01$ ) (Supplement Fig. $2 A)$ indicating that FK866- induced cell death did not depend on p53 function. Huh7 cells treated with FK866 [10 nM] for $72 \mathrm{~h}$ showed a 1.5fold increase in $\mathrm{An}^{+} / \mathrm{Pl}^{+}$cells compared to control cells $(\mathrm{p}<$ 0.05) (Fig. 2D) whereas the number of $\mathrm{An}^{+} / \mathrm{Pl}^{+} \mathrm{Hep} 3 \mathrm{~B}$ cells increased further $(+3.0$-fold, $p<0.01)$. Co-stimulation with NMN ameliorated the induction of cell death in Huh7 cells ( $p$ $1 / 40.09$ ) and completely rescued FK866- induced cell death in Hep3B cells $(p<0.01)$ (Fig. 2D).

\subsection{Dysregulation of the AMPK/mTOR signaling} pathway in hepatocarcinoma cells compared to non-cancerous hepatocytes

Growing evidence suggests that mTOR and AMPK dysregulation play an important role in hepatocellular carcinogenesis $[20,31]$. Therefore, we compared the protein amount of mTOR and its downstream target p70S6 kinase and also AMPKa activation in non-cancerous primary human hepatocytes and hepatocarcinoma cells. An increased protein level of total mTOR and p70S6 kinase was found in hepatocarcinoma cells compared to non-cancerous hepatocytes (Fig. 3A). In contrast, AMPKa activation was enhanced in non-cancerous primary human hepatocytes $(\mathrm{PHH})$ compared to Huh7 and Hep3B cells despite equal AMPKa total protein amount (Fig. 3A). This suggests that mTOR signaling and AMPKa activation are involved in metabolic adaptation of hep- atocarcinoma cells and might be interesting targets for prevention of cancer cell growth.

\subsection{FK866-induced energy stress activated AMPKa and led to inhibition of mTOR complex 1 signaling in hepatocarcinoma cells}

To test the efficacy of FK866-induced NAD depletion to activate AMPKa and inhibit the mTOR signaling pathway, we measured the phosphorylation state of different members of the AMPK/mTOR complexl cascade. FK866 treatment increased the phosphorylation of AMPKa at Thr172 (+3.3-fold, $p<0.01$ ) in hepatocarcinoma cells (Fig. 3B). This was associated with a significant down regulation of phosphorylated mTOR (Ser2448) by $-50.7 \pm 0.1 \%(p<0.05)$ and the phosphorylation of its down-stream target $70 \mathrm{~S}$ ribosomal protein S6 kinase (p70S6K) (by $-94.7 \pm 2.4 \%, p<0.001$ ) and eukaryotic initiation factor $4 \mathrm{E}$ binding protein 1 (4E-BP1)(by $-30.0 \pm$ $.0 .1 \%, p<0.05)$ indicating reduced protein synthesis and cell growth (Fig. 3B). Co-treatment with NMN [500 mM] completely reversed the FK866-induced effects on AMPKa activa- tion and mTOR complex 1 signaling inhibition suggesting that the NMN biosynthetic activity of NAMPT is relevant in mediating the effects of FK866. NMN alone had no impact on AMPKa activation and mTORC1 signaling in hepatocarcinoma cells (Fig. 3B). Non- cancerous human hepatocytes treated with equal amounts of FK866 for $48 \mathrm{~h}$ did not show significant changes in AMPKa acti- vation and mTOR phosphorylation (Supplement Fig. 1C) verifying their lower sensitivity to FK866.

\section{Discussion}

During malignant transformation the cellular metabolism undergoes multiple molecular and metabolic adaptations to support cell growth and survival. NAD is a key determinant in cancer cell biology as it is essential for redox reactions and key component of signaling pathways that regulate transcription, DNA repair, apoptosis and metabolism [1]. In mammals, NAMPT is a main regulator of the intracellular NAD pool $[2,3]$. Here, we investigated whether or not the NAMPT inhibitor, FK866, would affect intra- cellular NAD and ATP concentrations in hepatocarcinoma cells and consequently would be able to regulate the activity of the metabolic sensors AMPK and mTOR. Our study showed that FK866 rapidly reduced NAD levels in hepatocarcinoma cells and led to delayed ATP depletion which could be ameliorated by administration of NMN. Break down 
Published in final edited form as: Biochemical and Biophysical Research Communications 458 (2015) 334e340, http://dx.doi.org/10.1016/i.bbrc.2015.01.111

of ATP levels was associated with increased cell death. In contrast to another study [6], we demonstrated that FK866 reduced NAMPT activity, depleted NAD and ATP content and induced cell death in p53-deficient Hep3B cells suggesting that FK866-mediated cell death does not depend on functional p53. Our results are in line with a study performed in chronic lymphocytic leukemia cells [14]. In our study, especially Hep3B cells showed a high sensitivity to FK866 and an increased number of dead cells occurred already after $48 \mathrm{~h}$ of FK866 treatment. Interestingly, non- cancerous human hepatocytes subjected to the same FK866 treat- ment as hepatocarcinoma cells did not display reduced NAMPT activity and NAD content even at a FK866 concentration 10-fold of the EC50 (EC50 $8.2 \mathrm{nM}$ ) indicating a lower sensitivity of noncancerous cells to FK866. This has also been described for normal

blood cells $[6,7]$. Therefore, FK866 represents an interesting com- pound in cancer cell therapy as it progressively exhausts NAD content in cells with a high NAD turnover that mainly rely on nicotinamide and the NAMPT-mediated NAD salvage pathway as source of NAD. Cancer cells have a significantly higher NAD turn- over than normal cells to sustain their rapid proliferation, relative genomic instability, permanently ongoing DNA repair, increased aerobic glycolysis and increased activity of NAD-dependent deacetylases $[1,12,13]$. This is in line with results of our previous study showing that the expression of SIRT1, a NAD-dependent deacetylase, was significantly higher in hepatocarcinoma cells than in noncancerous hepatocytes [9].

In this study we could demonstrate that NAMPT inhibition by FK866 led to a sharp decline of intracellular ATP levels and there- fore induced energy stress. As a key physiological energy sensor, AMPK is a major regulator of cellular energy homeostasis that co- ordinates multiple metabolic pathways to balance energy supply [24]. Several studies have shown that AMPK activators exhibit inhibitory effects on cancer cell growth $[32,33]$. AMPK is known to phosphorylate and activate tuberous sclerosis complex (TSC)2, a negative regulator of mTOR [34]. Therefore, the AMPK/mTOR pathway serves as a signaling nexus for regulating cellular meta- bolism, energy homeostasis, and cell growth, and dysregulation of each pathway may contribute to the development of $\operatorname{HCC}[20,26]$. 
Published in final edited form as: Biochemical and Biophysical Research Communications 458 (2015) 334e340,

http://dx.doi.org/10.1016/i.bbrc.2015.01.111

A

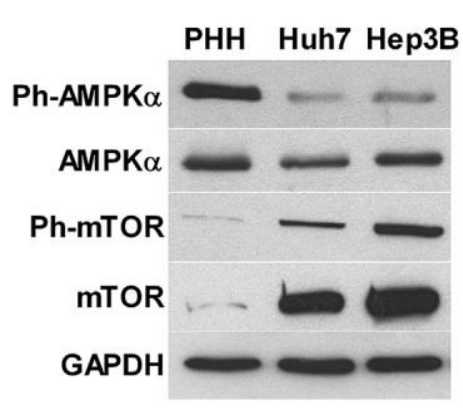

B

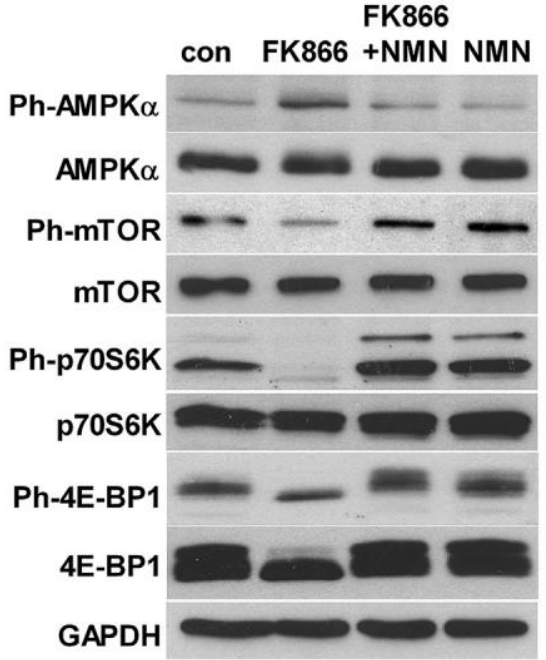

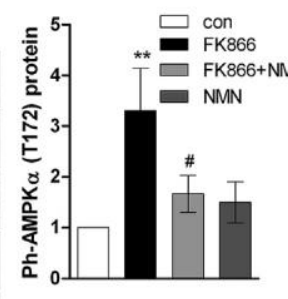
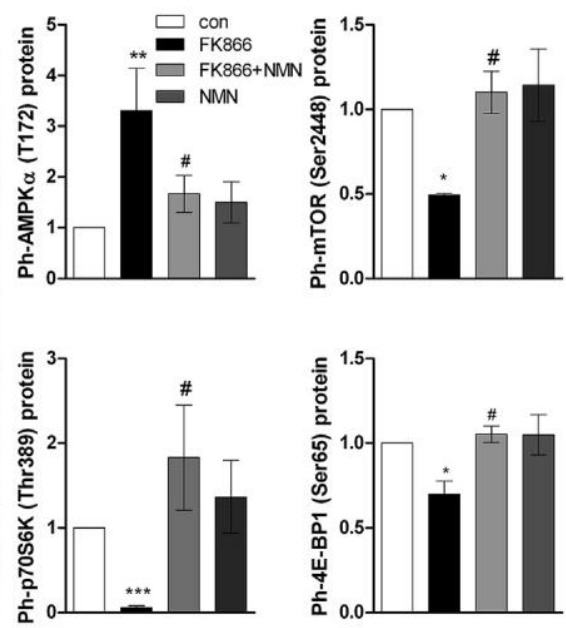

Fig. 3. Expression of mTOR and AMPK in hepatocarcinoma cells and non-cancerous human hepatocyes and its regulation by FK866. A) Western blot analysis of AMPK and mTOR expression in lysates of non-cancerous, primary human hepatocytes (PHH), Huh7 and Hep3B cells (n 1/4 3). B) Western blot analysis of the $\mathrm{AMPK} / \mathrm{mTORC} 1$ signaling pathway in lysates of Huh7 cells treated with FK866 [10 nM], a combination of FK866 [10 nM]+NMN [500 mM] or NMN [500 mM] alone for $48 \mathrm{~h}(\mathrm{n} 1 / 43)$. GAPDH was used as loading control. One representative blot out of 3 independent experiments is shown. Background-corrected densitometric values were normalized to control (serum-free medium). Data are represented as mean \pm SEM and statistical analysis was performed using one-way ANOVA and the Bonferroni post hoc test $\left({ }^{*} p<0.05,{ }^{* *} p<0.01,{ }^{* *} p<0.001\right.$ compared to serum-free medium control; \#p $<0.05, \# \#<0.01, \# \# p<0.001$ compared to FK866 [10 nM]).

Since the discovery that the mTOR pathway is hyperactivated in many cancers including $\mathrm{HCC}[25,26,31,35]$, there is a great interest in finding molecular pathways and novel compounds that target AMPK/mTOR signaling as novel treatment option for HCC. We could show that components of the mTORC 1 cascade were significantly higher expressed in hepatocarcinoma cells than in non-cancerous hepatocytes. Additionally, our data revealed that the activation of AMPKa was significantly decreased in hepatocarcinoma cells. Reduced AMPK activity has also been detected in primary human breast cancer [36] and lymphoma [21] cells. Thus, a dysregulated AMPK activity may represent an important regulatory step during tumor initiation and progression, allowing cancer cells to gain a metabolic growth advantage by enhancing aerobic glycolysis (Warburg effect) [21]. We made the intriguing discovery that FK866 acts as an AMPK activator in cancer cells potentially through its ability to induce cellular energy stress. Activation of AMPKa was associated with a down regulation of the mTORC1 pathway. All FK866 induced effects could be completely reversed by NMN suggesting that these effects were mediated by NAD. mTORC1 inhibi- tion led to decreased activation of its two downstream targets, p70S6K and 4E-BP1. p70S6K and 4E-BP1 are major regulators of protein translation and cellular growth [35]. This contradicts a study performed in neuronal cells where FK866 or a NAMPT knock down was shown to reduce AMPK activation [37]. However, this can be explained by the use of non-cancerous neuronal cells compared to cancer cells in our study.

In summary, our study showed the importance of the NAMPTmediated NAD salvage pathway for energy homeostasis in hep- atocarcinoma cells. Furthermore, FK866-induced NAMPT inhibition led to activation of AMPK and inhibition of mTOR signaling sug- gesting a putative use of FK866 alone or as a chemotherapeutic sensitizing drug to reduce cancer cell growth. In every case of po- tential therapeutic use, administration of NMN as antidote may be useful to modulate or counteract FK866 toxicity. Only early stages of HCC are curable with today's treatment protocols, therefore new therapeutic strategies are urgently needed and NAMPT inhibition represents a potential novel treatment approach.

\section{Conflict of interest}

All authors have nothing to disclose.

\section{Acknowledgments}

Primary human hepatocytes were kindly provided by the "Virtual Liver Network", a major national initiative on Systems Biology of the Liver. This work was supported by the German Federal Ministry of Education and Research (BMBF), "Virtual Liver 
Published in final edited form as: Biochemical and Biophysical Research Communications 458 (2015) 334e340, http://dx.doi.org/10.1016/j.bbrc.2015.01.111

Network", grant FKZ 0315753 to TSW. We thank our technician Sandy Richter for excellent work. We also gratefully thank Prof. Dr. Kurt Engeland (Molecular Oncology, Faculty of Medicine, University of Leipzig) for providing Huh7 cells and Prof.Dr.Sven-Erik Behrens (Institute of Biotechnology, MartinLuther-University Halle-Wit- tenberg) for providing Hep3B cells.

Appendix A. Supplementary data

Supplementary data related to this article can be found at http:// dx.doi.org/10.1016/i.bbrc.2015.01.111.

Transparency document

The transparency document associated with this article can
be found in the online version at

http://dx.doi.org/10.1016/j.bbrc.2015.01.111. 
Published in final edited form as: Biochemical and Biophysical Research Communications 458 (2015) 334e340,

http://dx.doi.org/10.1016/j.bbrc.2015.01.111

\section{References}

[1] A. Chiarugi, C. Dolle, R. Felici, et al., The NAD metabolomeea key determinant of cancer cell biology, Nat. Rev. Cancer 12 (2012) $741 \mathrm{e} 752$.

[2] J.R. Revollo, A.A. Grimm, S. Imai, The regulation of nicotinamide adenine dinucleotide biosynthesis by Nampt/PBEF/visfatin in mammals, Curr. Opin. Gastroenterol. 23 (2007) 164e170.

[3] C. Dolle, R.H. Skoge, M.R. Vanlinden, et al., NAD biosynthesis in human- seenzymes, metabolites and therapeutic aspects, Curr. Top. Med. Chem. 13 (2013) 2907 e2917.

[4] M. Rechsteiner, D. Hillyard, B.M. Olivera, Magnitude and significance of NAD turnover in human cell line D98/AH2, Nature 259 (1976) 695 e696.

[5] T.Q. Bi, X.M. Che, Nampt/PBEF/visfatin and cancer, Cancer Biol. Ther. 10 (2010) 119e125.

[6] B.K. Thakur, T. Dittrich, P. Chandra, et al., Involvement of p53 in the cytotoxic activity of the NAMPT inhibitor FK866 in myeloid leukemic cells, Int. J. Cancer 132 (2013) $766 \mathrm{e} 774$.

[7] M. Cea, A. Cagnetta, M. Fulciniti, et al., Targeting NAD+ salvage pathway induces autophagy in multiple myeloma cells via mTORC 1 and extracel- lular signal-regulated kinase (ERK1/2) inhibition, Blood 120 (2012) 3519e3529.

[8] A. Garten, S. Petzold, A. Barnikol-Oettler, et al., Nicotinamide phosphor- ibosyltransferase (NAMPT/PBEF/visfatin) is constitutively released from hu- man hepatocytes, Biochem. Biophys. Res. Commun. 391 (2010) 376 e381.

[9] S. Schuster, M. Penke, T. Gorski, et al., Resveratrol differentially regulates NAMPT and SIRT1 in hepatocarcinoma cells and primary human hepatocytes, PLoS One 9 (2014) e91045.

[10] B. Stewart, C.P. Wild, World Cancer Report 2014, WHO Press, 2014.

[1 1] J.M. Llovet, S. Ricci, V. Mazzaferro, et al., Sorafenib in advanced hepatocellular carcinoma, N. Engl. J. Med. 359 (2008) 378 e390.

[12] M. Hasmann, I. Schemainda, FK866, a highly specific noncompetitive inhibitor of nicotinamide phosphoribosyltransferase, represents a novel mechanism for induction of tumor cell apoptosis, Cancer Res. 63 (2003) 7436e7442.

[13] J.A. Khan, X. Tao, L. Tong, Molecular basis for the inhibition of human NMPRTase, a novel target for anticancer agents, Nat. Struct. Mol. Biol. $13(2006)$ 582e588.

[14] I. Gehrke, E.D. Bouchard, S. Beiggi, et al., On-target effect of FK866, a nico- tinamide phosphoribosyl transferase inhibitor, by apoptosismediated death in chronic lymphocytic leukemia cells, Clin. Cancer Res. 20 (2014) 4861 e4872.

[15] A. Nahimana, A. Attinger, D. Aubry, et al., The NAD biosynthesis inhibitor APO866 has potent antitumor activity against hematologic malignancies, Blood 113 (2009) 3276 e3286.

[16] T.Q. Bi, X.M. Che, X.H. Liao, et al., Overexpression of Nampt in gastric cancer and chemopotentiating effects of the Nampt inhibitor FK866 in combination with fluorouracil, Oncol. Rep. 26 (2011) 1251e1257.

[17] B. Tan, D.A. Young, Z.H. Lu, et al., Pharmacological inhibition of nicotinamide phosphoribosyltransferase (NAMPT), an enzyme essential for NAD+ biosyn- thesis, in human cancer cells: metabolic basis and potential clinical implica-tions, J. Biol. Chem. 288 (2013) 3500 e3511.

[18] M. Muruganandham, A.A. Alfieri, C. Matei, et al., Metabolic signatures asso-ciated with a NAD synthesis inhibitor-induced tumor apoptosis identified by $1 \mathrm{H}$-decoupled-31P magnetic resonance spectroscopy, Clin. Cancer Res. 11 (2005) 3503e3513.

[19] M.K. Christensen, K.D. Erichsen, U.H. Olesen, et al., Nicotinamide phosphor-ibosyltransferase inhibitors, design, preparation, and structureactivity rela- tionship, J. Med. Chem. 56 (2013) 9071 e9088.

[20] J. Cheng, T. Huang, Y. Li, et al., AMP-activated protein kinase suppresses the in vitro and in vivo proliferation of hepatocellular carcinoma, PLoS One 9 (2014) e93256.

[21] B. Faubert, G. Boily, S. Izreig, et al., AMPK is a negative regulator of the Warburg effect and suppresses tumor growth in vivo, Cell. Metab. 17 (2013) $113 e 124$.

[22] L. Zheng, W. Yang, F. Wu, et al., Prognostic significance of AMPK activation and therapeutic effects of metformin in hepatocellular carcinoma, Clin. Cancer Res. 19 (2013) 5372e5380.

[23] D.R. Bolster, S.J. Crozier, S.R. Kimball, et al., AMP-activated protein kinase suppresses protein synthesis in rat skeletal muscle through down-regulated mammalian target of rapamycin (mTOR) signaling, J. Biol. Chem. 277 (2002) 23977e23980.

[24] D.G. Hardie, The AMP-activated protein kinase pathwayenew players up-stream and downstream, J. Cell. Sci. 117 (2004) 5479 e5487.

[25] L. Zhou, Y. Huang, J. Li, et al., The mTOR pathway is associated with the poor prognosis of human hepatocellular carcinoma, Med. Oncol. 27 (2010) $255 \mathrm{e} 261$.

[26] R.E. Ashworth, J. Wu, Mammalian target of rapamycin inhibition in hepato- cellular carcinoma, World J. Hepatol. 6 (2014) $776 \mathrm{e} 782$.

[27] F. Chiarini, C. Evangelisti, J.A. McCubrey, et al., Current treatment strategies for inhibiting mTOR in cancer, Trends Pharmacol. Sci. 36 (2) (2015) 124e135.

[28] T.S. Weiss, S. Pahernik, I. Scheruebl, et al., Cellular damage to human hepa- tocytes through repeated application of 5-aminolevulinic acid, J. Hepatol. 38 (2003) $476 \mathrm{e} 482$.

[29] G.C. Elliott, J. Ajioka, C.Y. Okada, A rapid procedure for assaying nicotinamide phosphoribosyltransferase, Anal. Biochem. 107 (1980) $199 \mathrm{e} 205$.

[30] W. Xu, Y. Li, C. Liu, et al., Protein lysine acetylation guards metabolic ho- meostasis to fight against cancer, Oncogene 33 (2014) $2279 \mathrm{e} 2285$.

[31] A. Villanueva, D.Y. Chiang, P. Newell, et al., Pivotal role of mTOR signaling in hepatocellular carcinoma, Gastroenterology 135 (2008) $1972 \mathrm{e} 1983$.

[32] J. Sinnett-Smith, K. Kisfalvi, R. Kui, et al., Metformin inhibition of mTORC1 activation, DNA synthesis and proliferation in pancreatic cancer cells: dependence on glucose concentration and role of AMPK, Biochem. Biophys. Res. Commun. 430 (2013) 352 e357.

[33] J. Woodard, L.C. Platanias, AMP-activated kinase (AMPK)-generated signals in malignant melanoma cell growth and survival, Biochem. Biophys. Res. Com- mun. 398 (2010) 135e139. 
Published in final edited form as: Biochemical and Biophysical Research Communications 458 (2015) 334e340, http://dx.doi.org/10.1016/j.bbrc.2015.01.111

[34] K. Inoki, T. Zhu, K.L. Guan, TSC2 mediates cellular energy response to control cell growth and survival, Cell 115 (2003) 577 e590.

[35] D.M. Sabatini, mTOR and cancer: insights into a complex relationship, Nat. Rev. Cancer 6 (2006) 729e734.

[36] S.M. Hadad, L. Baker, P.R. Quinlan, et al., Histological evaluation of AMPK signalling in primary breast cancer, BMC Cancer 9 (2009) 307.

[37] P. Wang, T.Y. XU, Y.F. Guan, et al., Nicotinamide phosphoribosyltransferase protects against ischemic stroke through SIRT1-dependent adenosine monophosphate-activated kinase pathway, Ann. Neurol. 69 (2011) 360e374 